BMJ Open Sport \& Exercise Medicine

\title{
Clinical outcomes of arthroscopic repair of acetabular labral tears
}

\section{Carlos César Vassalo, ${ }^{1}$ Antônio Augusto Guimarães Barros, ${ }^{1}$ Lincoln Paiva Costa, Euler de Carvalho Guedes, ${ }^{1}$ Marco Antônio Percope de Andrade ${ }^{2}$}

\section{ABSTRACT \\ Purpose To evaluate the primary clinical outcomes of arthroscopic labral repair.}

Methods All patients who underwent arthroscopic repair of the acetabular labrum performed by a senior surgeon between October 2010 and December 2013 were invited to participate in this prospective study. Patients included were those who had a preoperative diagnosis of labral tears, a lateral centre edge greater than $25^{\circ}$ and a labral tear believed to be suturable during the intraoperative evaluation. Patients with Tönnis grade 2 or grade 3 hip osteoarthritis and those who had undergone a previous hip surgery were excluded. All patients were evaluated using the modified Harris Hip Score (mHHS) during the final appointment before surgery, 4 months after surgery and at the final evaluation. Interviews were conducted by the senior surgeon.

Results Eighty-four patients (90 hips) underwent arthroscopic repair. The mean age was 44.2 years and the mean follow-up period was 43.0 months (minimum of 25 months and maximum of 59 months). The mean mHHS was 80.4 preoperatively, 95.0 at 4 months postoperatively and 96.6 at final evaluation. A statistically significant difference existed among these scores $(p<0.001)$.

Conclusion Arthroscopic labral repair was associated with a clinically significant improvement in $\mathrm{mHHS}$ after short-term (4 months) and medium-term (43 months) follow-up.

Level of evidence Level IV, therapeutic case series.

\section{INTRODUCTION}

Hip labral tears are a source of hip pain, occur mainly in young adults and are most often associated with femoroacetabular impingement (FAI). ${ }^{1}$ FAI is a dynamic condition caused by a change in the hip morphology at the femoral side (cam-type impingement), acetabular side (pincer-type impingement) or both. A restricted range of movement caused by repetitive impingement between the femoral head and the acetabular rim results in acetabular labrum and articular cartilage lesions and predisposes patients to early joint degeneration. ${ }^{2}$ The severity of labral and chondral lesions may progress until they are treated; therefore, early diagnosis and treatment are important. ${ }^{45}$

Arthroscopy is a treatment option for FAI and associated labral tears. It presents

\section{What are the new findings?}

In patients with a mean age of 44 years, arthroscopic labral repair was associated with a clinically significant improvement in self-reported hip pain and function (modified Harris Hip Score) at both short-term (4 months) and medium-term (43 months) follow-up.

\section{How might it impact on clinical practice?}

Patients can expect an improvement in self-reported hip function by 4 months postoperatively.

low complication rates and good clinical outcomes, especially in cases where severe joint damage is absent. ${ }^{6-11}$ The surgical procedure aims to treat labral pathology and correct FAI, restoring the labrum anatomy and joint-sealing function, thus decreasing pain and possibly preventing progression to osteoarthritis. $^{1213}$

The purpose of this study was to evaluate the primary clinical outcomes of arthroscopic labral repair. We hypothesised that labral repair results in sustained, medium-term, clinical improvement in patients.

\section{METHODS}

All patients who underwent arthroscopic repair of the acetabular labrum performed by a senior surgeon (more than 500 hip arthroscopies of experience) between October 2010 and December 2013 were invited to participate in this prospective study. Patients included were those who had a preoperative diagnosis of labral tears, a lateral centre edge greater than $25^{\circ}$ and a labral tear believed to be suturable during the intraoperative evaluation. Patients with Tönnis grade 2 or grade 3 hip osteoarthritis, those who had undergone a previous hip surgery and those who refused to sign the informed consent were excluded.

Preoperative diagnosis was based on clinical examination and imaging findings. Imaging examinations included anteroposterior, 
false-profile, frog lateral and $45^{\circ}$ Dunn radiographs of the affected hip joint, along with MRI or magnetic arthro-resonance with gadolinium. ${ }^{14}{ }^{15}$ Surgery was indicated after failure of conservative treatments that included moderate rest, change of sport activity, non-steroidal anti-inflammatory drugs and attendance at physical therapy appointments for at least 3 months.

Procedures were performed under general anaesthesia combined with a regional block. Patients were placed in the supine position on an orthopaedic traction table, with hip-joint traction. Two portals were created: an anterolateral portal to access the joint and a mid-anterior one performed under direct visualisation. The bone changes that caused impingement were corrected, and cartilage lesions were treated by debridement and microfracture.

Labral suture was performed during the procedure only on patients with sufficient healthy tissue to restore joint-sealing function and those without extensive chondral lesions; otherwise, degenerated labral rims were debrided and trimmed, and the patients were excluded from the study. For labral sutures, $2.7 \mathrm{~mm}$ anchors were placed at every $1 \mathrm{~cm}$ of damaged labrum and positioned $2-3 \mathrm{~mm}$ from the acetabular rim to avoid penetrating the joint surface. The labrum was repaired with a loop or translabral suture, and the suture knot was secured in the direction of the labrum capsular side. After fixation, traction was removed, and restoration of the labrum joint-sealing function was assessed. The mean procedure time was $99 \mathrm{~min}$, and the mean traction time during the procedure was $75 \mathrm{~min}$. In most cases patients remained in hospital overnight as this was the standard institution protocol. On average, patients were discharged from the hospital 21 hours after the procedure (ranging from 16 to 24 hours). Postoperative rehabilitation was based on a four-stage protocol focusing on patients' return to normal activities, as described by Wahoff and Ryan. ${ }^{16}$

All patients were evaluated using the modified Harris Hip Score (mHHS). Questionnaires were completed by patients during the final appointment before surgery, 4 months after surgery and at the final evaluation. Outcome interviews were all conducted by the senior surgeon.

\section{Statistical analysis}

Descriptive measures (mean and SD, median, and first and third quartiles) were used for the quantitative variables and frequency distributions for the qualitative ones. The Friedman non-parametric test was used to assess differences in mHHS values between preoperative and postoperative periods ( 4 months and the final evaluation) because assumption of data normality was violated according to the Shapiro-Wilk test. Friedman test was applied because mHHS was compared over time on the same patient (preoperatively, at 14 months after surgery and at final evaluation). The test aimed to determine whether any study period had a greater effect on the results. In cases with significant differences among the three study periods, a comparison between two time points was made using the Wilcoxon test. Study data
Table 1 Demographic information of the 84 study patients

\begin{tabular}{lc} 
Demographic characteristics/variables & \\
\hline Patients, $\mathrm{n}$ & 84 \\
Sex, $\mathrm{n}(\%)$ & \\
$\quad$ Male & $32(61.9)$ \\
$\quad$ Female & $32(38.1)$ \\
Mean age, years (SD) & $44.2(10.3)$ \\
Side, $\mathrm{n}$ (\%) & \\
Left hip & $47(52.2)$ \\
Right hip & $31(34.4)$ \\
\hline Both hips & $12(13.4)$ \\
Number of anchors used, $\mathrm{n}(\%)$ & \\
\hline 1 & $44(48.9)$ \\
2 & $35(38.9)$ \\
3 & $8(8.9)$ \\
4 & $2(2.2)$ \\
5 & $1(1.1)$ \\
Mean follow-up period, months (SD) & $43.0(9.4)$ \\
\hline
\end{tabular}

were analysed using Predictive Analytics Software (V.18). Significance level was set at 5\% in all statistical tests. Associations were considered statistically significant for $\mathrm{p}$ values $\leq 0.05$.

\section{RESULTS}

During the study period, 89 patients met the inclusion criteria, and a total of 95 hip arthroscopy procedures were performed. Four patients $(4.4 \%)$ were excluded from the final evaluation because their condition progressed to total hip arthroplasty due to persistent pain. One patient was lost to follow-up. Therefore, the sample consisted of 84 patients with a total of 90 arthroscopy procedures; these included 47 performed on the left hip (52.2\%), 31 on the right hip $(34.4 \%)$ and 6 performed bilaterally $(13.4 \%)$.

Thirty -two patients were female $(38.1 \%)$ and 52 were male $(61.9 \%)$. Labral tear was repaired with only one anchor in 44 patients $(48.9 \%)$, two anchors in 35 $(38.9 \%)$, three anchors in $8(8.9 \%)$ and more than three anchors in 3 (3.3\%). Patients' age ranged from 23 to 67 years, with a mean age of 44.2 years $(\mathrm{SD}=10.3)$. The mean follow-up period was 43.0 months, ranging from 25 to 59 months (table 1 ).

A comparative analysis of mHHS of the total sample over time using the Friedman test indicated a significant difference between the three study periods $(p<0.001$; table 2). Multiple comparisons were made using the Friedman test. Because the null hypothesis was rejected, at least one study period was shown to differ from the others. A multiple comparison test was performed to determine which period was different. The Wilcoxon test determined the differences between group pairs (table 3). 
Table 2 Evolution of modified Harris Hip scores over time

\begin{tabular}{llll}
\hline Variable & Study periods & Mean (SD) & P values \\
\hline Modified Harris & Before surgery & $80.4(7.2)$ & $<0.001$ \\
Hip Score & 4 months after & $95.0(6.3)$ & \\
& $\begin{array}{l}\text { surgery } \\
\text { Final evaluation }\end{array}$ & & \\
& & & \\
&
\end{tabular}

The $p$ values were obtained using the Friedman test.

The mean preoperative mHHS was $80.4 \pm 7.2$, whereas the postoperative (at 4 months) mHHS was 95.0 06.3 . The difference between these periods was statistically significant $(\mathrm{p}<0.001)$. In addition, a statistically significant difference existed between the mean preoperative mHHS values (80.4) and those at the final evaluation $(96.6 \pm 4.5) \quad(\mathrm{p}<0.001)$. With respect to the two postoperative periods, the mean mHHS at the final evaluation (96.6) was significantly higher than that at 4 months after the procedure $(\mathrm{p}<0.001$; table 3$)$.

\section{DISCUSSION}

Arthroscopic labral tear repair was associated with decreased pain and improved patient function by self-report at short-term and medium-term follow-up periods. mHHS was higher at 4 months after surgery (95.0 \pm 6.3$)$ than preoperatively $(80.4 \pm 7.2)$, and it increased after an average of 3.6 years $(96.6 \pm 4.5)$. These differences were all statistically significant. Four patients $(4.4 \%)$, during the study, required total hip arthroplasty.

Regarding treatment types for labral tears, studies have indicated that labral repair results in better mHHS values and greater reduction of degenerative changes than labral debridement. ${ }^{81718}$ Schilders et $a l^{18}$ compared arthroscopic treatments of labral tears in the presence of FAI. Ninety-six patients (101 hips) were included in the study; labral repair was performed in 69 hips and labral resection in 32 . The mHHS values in the preoperative and postoperative periods (ie, at the 2.4-year mean follow-up period) were used to assess the results. In the group subjected to labral suture, the mean preoperative mHHS was 60.2, which increased to 93.6 at the final evaluation. The mean score in this group was 7.3 points higher than that in the group subjected to labrum resection. This difference was statistically significant and indicated better outcomes for patients whose labra were preserved.

Similarly, Larson et $a l^{19}$ compared the arthroscopic results of debridement and labral repair in cases of FAI and observed better results with repair. There were 44 hips in the debridement group with a mean age of 32 years, and 50 hips in the repair group with a mean age of 28 years. The mean $\mathrm{mHHS}$ of the repair group was 64.5 before surgery and 94.3 in the final evaluation, which was 9.4 points higher than that in the debridement group. In our study, the acetabular labrum was preserved whenever possible, particularly in patients with a few cartilage lesions, considering that labrum is essential for joint-cartilage protection.

Byrd and Jones ${ }^{20}$ conducted a prospective evaluation of 37 consecutive patients (38 hips) who underwent an arthroscopic labrum repair (no patient was excluded from the study). The authors used mHHS to assess outcomes within a 2-year follow-up period. The mean mHHS increased 18.9, varying from 70.5 to 89.4. Patients with chondral lesions or acetabular dysplasia were included in the study. There were no complications; however, $11 \%$ of their patients required revision surgery. Our study showed an increase of 16.2 points in mHHS, similar to that reported by Byrd and Jones. ${ }^{20}$ However, the mean final score obtained by them was lower, probably because they included patients with chondral lesions and acetabular dysplasia.

Dippmann et $a t^{21}$ also evaluated arthroscopic labral repair for FAI, by $\mathrm{mHHS}$ and the pain visual analogue scale (VAS), in 87 patients. They observed a statistically significant increase in mHHS and an improvement in the VAS between 3 and 6 months after the procedure and no difference between 6 and 12 months. Our study showed a statistically significant difference in mHHS between 4 months (95.0) and the final evaluation (96.6), which means that patients improved over time.

Other papers with different functional scores have also demonstrated satisfactory labral repair outcomes. Jackson et $a l^{22}$ evaluated 54 patients with a mean age of 28.8 years who had undergone arthroscopic labral repair. The authors used four functional scores, VAS and patient satisfaction. They observed significant improvement in the four scores 2.4 years after the procedure; the mean mHHS was 63.7 preoperatively and 89.9 at the final evaluation. Furthermore, $85.2 \%$ patients had good or excellent results, which is in line with our results. However, only mHHS was used in our study.

Age and osteoarthritis may affect treatment of labral lesions. McCormick et $a l^{10}$ analysed the effect of these variables on outcome in 98 patients with a mean age of 40.9 years and a minimum follow-up period of 2 years.

\begin{tabular}{|c|c|c|c|}
\hline Variable & Follow-up periods & & $P$ values \\
\hline \multirow[t]{3}{*}{ Modified Harris Hip Score } & 80.4 (preoperative) & 95.0 (4 months after surgery) & $<0.001$ \\
\hline & & 96.6 (final evaluation) & $<0.001$ \\
\hline & 95.0 (4 months) & 96.6 (final evaluation) & $<0.001$ \\
\hline
\end{tabular}

The $p$ values were obtained using the Wilcoxon test. 
Assessed variables were age over 40 years and presence of a grade 4 chondral lesion (Outerbridge classification). After a mean follow-up period of 4.3 years, outcomes in patients aged $>40$ years and with signs of osteoarthritis were worse than in healthy younger patients. The mean age in our study was 44.2 years, which apparently had no impact on the results, but patients with severe joint-cartilage lesions were not included. Therefore, according to our study, age is not an isolated factor of poor prognosis for arthroscopic labral repair and the degree of cartilage damage should be considered more important for outcome. The strengths of this study include the relatively large sample of patients operated on an arthroscopic labral repair by the same surgeon and with a longer follow-up period than most studies in this area.

We report five study limitations in addition to this being a case series, a lower level of evidence than a controlled trial. Our study design does not allow us to comment on how the procedure of labral debridement would have performed on these patients. Second, although the presence of cartilage lesions negatively affects the results of arthroscopic labral repair, the degree of joint damage observed by arthroscopy was not quantified. A better characterisation of joint integrity could reveal whether patients with less significant improvement had hips with worse joint damage. Third, this study did not include in the analysis the presence of iatrogenic lesions that occurred during portal creation. This type of lesions could impair the outcomes of some patients. Fourth, all interviews were conducted by the senior surgeon and response bias to the surgeon was found in other studies. ${ }^{23}$ Finally, questionnaires such as the Non-Arthritic Hip Score and the Hip Outcome Score could have been employed to measure the outcomes, but only mHHS was used .

\section{CONCLUSION}

Arthroscopic labral repair was associated with a clinically significant improvement in mHHS after short-term (4 months) and medium-term (43 months) follow-up.

\section{Competing interests None declared.}

\section{Patient consent Obtained.}

Ethics approval The study was approved by the research ethics committee of our institution (Comitê de Ética do Hospital Madre Teresa via plataforma Brasil-Governo Federal do Brasil).

Provenance and peer review Not commissioned; externally peer reviewed.

Open Access This is an Open Access article distributed in accordance with the Creative Commons Attribution Non Commercial (CC BY-NC 4.0) license, which permits others to distribute, remix, adapt, build upon this work non-commercially, and license their derivative works on different terms, provided the original work is properly cited and the use is non-commercial. See: http://creativecommons.org/ licenses/by-nc/4.0/ (c) Article author(s) (or their employer(s) unless otherwise stated in the text of the article) 2018. All rights reserved. No commercial use is permitted unless otherwise expressly granted.

\section{REFERENCES}

1. Beaulé PE, O'Neill M, Rakhra K, et al. Acetabular labral tears. J Bone Joint Surg Am 2009;91:701-10.

2. Parvizi J, Leunig M, Ganz R. Femoroacetabular impingement. J Am Acad Orthop Surg 2007;15:561-70.

3. Ganz R, Parvizi J, Beck M, et al. Femoroacetabular impingement: a cause for osteoarthritis of the hip. Clin Orthop Relat Res 2003;417:112-20.

4. Burnett RS, Della Rocca GJ, Prather H, et al. Clinical presentation of patients with tears of the acetabular labrum. J Bone Joint Surg Am 2006;88:1448-57.

5. Johnston TL, Schenker ML, Briggs KK, et al. Relationship between offset angle alpha and hip chondral injury in femoroacetabular impingement. Arthroscopy 2008;24:669-75.

6. Byrd JW, Jones KS. Arthroscopic management of femoroacetabular impingement: minimum 2-year follow-up. Arthroscopy 2011;27:1379-88.

7. Krych AJ, Thompson M, Knutson Z, et al. Arthroscopic labral repair versus selective labral debridement in female patients with femoroacetabular impingement: a prospective randomized study. Arthroscopy 2013;29:46-53.

8. Larson CM, Giveans MR. Arthroscopic debridement versus refixation of the acetabular labrum associated with femoroacetabular impingement. Arthroscopy 2009;25:369-76.

9. Dietrich F, Ries C, Eiermann C, et al. Complications in hip arthroscopy: necessity of supervision during the learning curve. Knee Surg Sports Traumatol Arthrosc 2014;22:953-8.

10. McCormick F, Nwachukwu BU, Alpaugh K, et al. Predictors of hip arthroscopy outcomes for labral tears at minimum 2-year follow-up: the influence of age and arthritis. Arthroscopy 2012;28:1359-64.

11. Philippon MJ, Briggs KK, Yen YM, et al. Outcomes following hip arthroscopy for femoroacetabular impingement with associated chondrolabral dysfunction: minimum two-year follow-up. J Bone Joint Surg Br 2009;91:16-23.

12. Kelly BT, Weiland DE, Schenker ML, et al. Arthroscopic labral repair in the hip: surgical technique and review of the literature. Arthroscopy 2005;21:1496-504.

13. Groh MM, Herrera J. A comprehensive review of hip labral tears. Curr Rev Musculoskelet Med 2009;2:105-17.

14. Czerny C, Hofmann S, Neuhold A, et al. Lesions of the acetabular labrum: accuracy of MR imaging and MR arthrography in detection and staging. Radiology 1996;200:225-30.

15. Plötz GMJ, Brossmann J, Schünke M, et al. Magnetic resonance arthrography of the acetabular labrum. J Bone Joint Surg Br 2000;82-B:426-32.

16. Wahoff M, Ryan M. Rehabilitation after hip femoroacetabular impingement arthroscopy. Clin Sports Med 2011;30:463-82.

17. Kemp JL, Collins NJ, Makdissi M, et al. Hip arthroscopy for intraarticular pathology: a systematic review of outcomes with and without femoral osteoplasty. Br J Sports Med 2012;46:632-43.

18. Schilders E, Dimitrakopoulou A, Bismil Q, et al. Arthroscopic treatment of labral tears in femoroacetabular impingement: a comparative study of refixation and resection with a minimum twoyear follow-up. J Bone Joint Surg Br 2011;93:1027-32.

19. Larson CM, Giveans MR, Stone RM. Arthroscopic debridement versus refixation of the acetabular labrum associated with femoroacetabular impingement: mean 3.5-year follow-up. Am J Sports Med 2012;40:1015-21.

20. Byrd JW, Jones KS. Prospective analysis of hip arthroscopy with 2-year follow-up. Arthroscopy 2000;16:588-92.

21. Dippmann C, Thorborg K, Kraemer O, et al. Hip arthroscopy with labral repair for femoroacetabular impingement: short-term outcomes. Knee Surg Sports Traumatol Arthrosc 2014;22:744-9.

22. Jackson TJ, Hanypsiak B, Stake CE, et al. Arthroscopic labral base repair in the hip: clinical results of a described technique. Arthroscopy 2014;30:208-13.

23. McGrory BJ, Morrey BF, Rand JA, et al. Correlation of patient questionnaire responses and physician history in grading clinical outcome following hip and knee arthroplasty. A prospective study of 201 joint arthroplasties. J Arthroplasty 1996;11:47-57. 